\title{
Humans: the missing link in manufacturing simulation?
}

\section{Tim Baines, Stephen Mason, Peer-Olaf Siebers and John Ladbrook}

\section{Introduction}

Organisations face constant pressure to improve products, facilities, technologies, methods, and work practices, and hence, the design and redesign of manufacturing systems is an on-going and complex task. Industrial environments are designed and created by engineers who use a combination of judgement, bargaining and analysis to assess the designs they create. However, although the theoretical steady state performance of a system can be easily calculated, interaction of resources and the time dependency of activities means that the dynamic behaviour of a system is much more difficult to determine. Computer based simulation enables systems to be modelled, and so allows better predictions of performance.

Computer simulations have traditionally focussed on the technological aspects of systems (e.g. machines, conveyors), and represented these with deterministic and stochastic data [2]. Such simulations however, frequently overestimate the production capacity of manufacturing systems. This can cause serious problems when the proposed system is implemented, and then fails to meet expectations. Our view is that the difference between predicted and actual performance is largely due to these models failing to adequately incorporate some key relationships. One such omission is the relationship between the performance of a person and factors impacting on this performance. This is particularly apparent when modelling manufacturing systems that have a high proportion of manual operations [1]. Hence to improve the accuracy of simulation it is necessary to realistically represent people, their behaviour and subsequent performance.

The research described in this paper has set out to illustrate the possible extent of error that occurs in manufacturing system simulation models due to poor representation of people. To do this, two contemporary models of human performance are first identified. These models represent performance reduction due to ageing, and performance variation caused by biorhythms. These models have then been incorporated in a discrete event simulation (DES) model of a manufacturing system, and subsequent execution of the models helps to illustrate the benefits of representing human behaviour more precisely in a typical simulation model.

The paper is structured as follows. Section 2 provides the background to this research, explaining the challenge in greater detail. Section 3 outlines the aim of the work and the method employed. Section 4 outlines the models of human factors, while Section 5 describes the implementation of these into a DES model. Section 6 presents the results from experimentation, following which conclusions are drawn.

\section{Simulation within a process of manufacturing system design}

Simulation is a technique of constructing a model that describes the behaviour of a real world system, and the resulting model can then be used to test how the performance of a proposed system alters over differing operating conditions. Discrete event simulation appears to be the standard tool used in the design of different automotive manufacturing systems [6]. DES is concerned with modelling a system by a representation in which state variables change instantaneously at separate points in time, directly associated with the beginning and end of events within a system [9].

Witness [8] typifies simulation software currently available to model manufacturing systems. Such tools are very well suited to modelling the technological component of a manufacturing facility. For example the "bath tub" distribution is usually incorporated as a standard feature for modelling the probability of machine failure [9]. Such a distribution is well accepted as being appropriate for representing machine breakdown behaviour. Conversely, the human element of a manufacturing system is poorly supported. People are treated as a pseudo-technological element and 
expected to behave in much the same fashion as an item of equipment. In practice, people's behaviour is far from this, and this could help to explain why simulation models do not model reality as well as could be expected.

The resource element concept is limited in its application for modelling human behaviour and performance. This problem is especially acute when modelling a system with a high proportion of manual work, such as an assembly line. Humans conducting such tasks do not lend themselves to simulation in the same way as machines; they are inherently unstable, unpredictable, and capable of independent action. For example, the performance of an individual will fluctuate depending on such factors as their ability, training and education, along with their physiological and psychological states and traits [3]. In addition, the physical and organisational environment created by the designer will also affect their performance.

Generally engineers are largely unaware of the social, psychological and physiological behaviours and needs of the workforce with which they interact. This is despite the fact that the social sciences contain an inordinate amount of information about workers, their behaviours and their lifestyles, which could be applied to improve the process of manufacturing system design. There are many reasons why manufacturing has not assimilated social science knowledge e.g. differences in language and methods and lack of predictive empirical relationships. The inclusion of human performance models in DES provides an opportunity to make system designers aware of both the importance and the impact of human factors.

To apply human performance modelling to DES requires both valid models of human factors and rigorous techniques. These elements, however, do not currently exist in the manufacturing arena. Models and techniques are emerging within the military and social science domains that clearly indicate valid modelling of worker performance is possible [13]. Hence, one solution to the problem of developing HPM capabilities within DES is to examine the work of the social sciences, and attempt to incorporate valid models into manufacturing simulation packages.

\section{Aim and method}

The aim of the work described in this paper has been to investigate the feasibility and impact of incorporating existing models of human performance into a typical DES model. Here, our intention has not been to suggest an idealised form of human performance model, but rather to explore and illustrate the extent to which the performance of a quite simple simulation can be altered by the inclusion of such models. To realise this aim, the methodology has been quite straightforward. First, it has been necessary to identify a typical simulation model that contains a variety of human and technological components. This has been achieved by selecting an assembly line model that has a relatively large number of manual operations, yet is not overly complicated with control rules and interdependencies.

The model selected was of the after test dress area of an automotive engine assembly line. This is the last section of the line prior to shipping and so is isolated from downstream disturbance. It is also isolated from upstream disturbance as the previous activities are well balanced. Similarly, a repair loop is sited just before the area so virtually no damaged or incomplete engines enter the dress area. Lastly, it is a small and thus manageable part of the line with only nine manual operations. A Witness model of the area had already been developed during the system design process. This then formed the test-bed simulation.

The second step has been to identify a number of human performance models to incorporate within the test-bed simulation. This has been achieved through a review of the literature, and two specific models have been identified which relate human factors to changes in manual cycle times, described in detail in Section 4. The first model combines current estimates of the performance decrement caused by ageing with estimates of the mediating effects of training and experience. The second model defines how performance alters over the period of a day due to biorhythms, also known as circadian rhythms. These have then been incorporated into the test model. A series of simulation runs have been conducted to assess the sensitivity of the simulation to the models. Section 5 presents the results of these experiments. 


\section{Selection and description of the human performance models}

This section describes the selection of the human performance models, along with the subsequent models chosen.

Selection of the models was based on three criteria. Firstly, the models should be valid in the context in which they were originally derived. Secondly, there should be sufficient literature to indicate that the factor represented by the model would be present in a manufacturing context. Thirdly, that the inputs required for the models should be easy to obtain. It was also desirable that the models would consist of mathematical functions.

Generally, two types of human performance models can be identified in the literature. Model of high-level factors usually deal with complex interactions of psychological mechanisms. For example job satisfaction is known to impact work performance, but it is mediated by psychological and environmental factors [5]. Such models are inherently complex, context specific and dependent on individual differences between people. The combination of low contextual validity and complexity makes such models unsuitable for manufacturing simulation.

Models of low-level factors represent basal physiological mechanisms. For example models of dehydration provide estimates of performance changes, mediated by environmental conditions [20]. In contrast to high-level models, such models are relatively simple and can be applied to any individual. These models are hence particularly suited to simulation. The two models selected represent low-level basal factors. The first represents performance changes caused by ageing, and the second represents the effects of daily biological rhythms.

\subsection{Human performance model 1: age related performance theory}

Much evidence exists to suggest that performance decreases with increasing age [7] and [18]. Both cognitive and physical faculties display a decrease in maximal performance of approximately $1 \%$ per year of age, after the age of about 20 [15]. Up to 20 years of age the human body is still developing, and performance increases until this "over the hill" point, which varies between individuals. Basic cognitive functions of the human body such as short term memory, processing speed, and decision making as well as gross physical faculties such as aerobic fitness, oxygen uptake, and maximal muscle strength have been shown to decrease in a linear fashion when considered in isolation [12] and [17]. These effects are all mediated by, and can be significantly reduced by, increases in technical and interpersonal skills, experience, physical fitness training, lifestyle and the development of personal coping mechanisms [4] and [16].

According to Warr [21], these effects mask the decrease in performance due to ageing. In an attempt to represent such masking mechanisms a model was developed based on the assumption that where a task requires significantly less than maximal performance the effective change in performance is not linear. The model assumes that age decrements are not measurable until after age 30 and that masking effects linearly reduce the impact of ageing until age 65 when the full impact of age is felt. It was assumed that the rate of performance decrement due to age (d) is not a constant $1 \%$ per year of age, but increase linearly from $0 \%$ at age 30 , to $1 \%$ at age 65 . Hence, for the age range $30-65, d$ is given by

$$
d=\frac{(a-30)}{35}
$$

where $d=$ rate of change of performance decrement due to age (\%); a=age in years.

The performance decrement at any age a is then given by

$D=d(a-30)$

where $D=$ performance decrement due to age (\%).

Eq. (4.2) provides a percentage decrease in performance, which can be applied directly to the DES test model to increase the cycle times at each station that is reliant on labour. The effects of this model are shown in Fig. 1. 


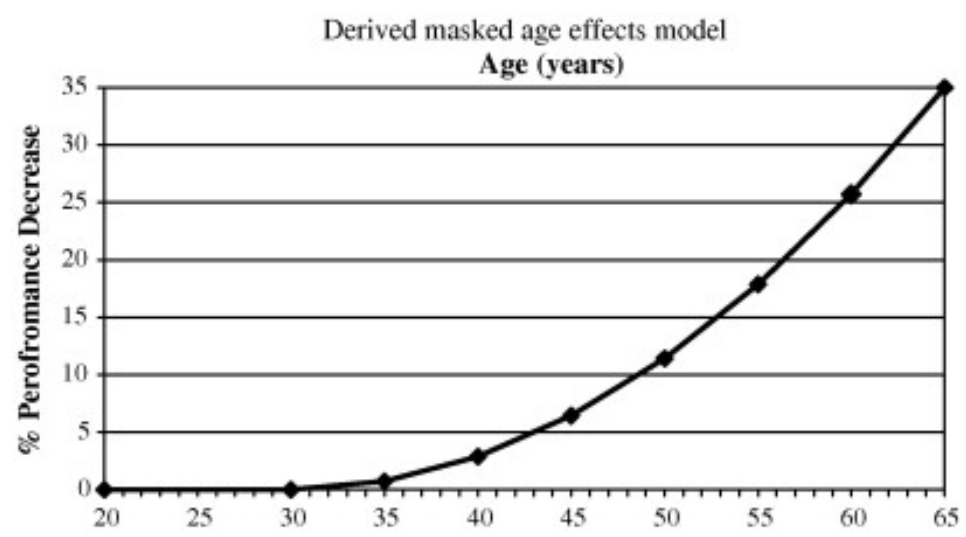

Fig. 1. - Graph of age related performance model output.

\subsection{Human performance model 2: circadian rhythm related performance theory}

Many of our processes, from basal systems such as hormone production, digestion, and homeostasis, to higher-level systems like cognition and locomotion display variations in arousal and efficiency over time [11]. Some, like the circadian rhythm $(C R)$, vary with a relatively simple function with a period of $24 \mathrm{~h}$, while others show more complex variations over weeks, months and even years. All the rhythms perform vital tasks of bio-regulation, and when allowed to perform their tasks unhindered the human body is allowed to maintain normal levels of exertion, effort, motivation, and physical and mental health. When the rhythms are disrupted, however, performance is degraded and health can be affected sufficiently to cause chronic and acute illnesses, such as cardiovascular problems, cancer, and clinical depression [10] and [14].

The main function of the CR is to prepare our bodies for sleep, through hormonal regulation of our most basic functions. At a certain time in the evening, typically over a range from 10:30 pm to 1:00 am, our bodies naturally enter sleep. This is usually accompanied by a reduction in core temperature, an increase in melatonin (the "sleep" hormone) and characteristic brain wave activity. The CR then causes us to wake after an average sleep duration of just over $7 \mathrm{~h}$. The actual preferred sleep and wake times vary between individuals. Although the existence of $C R$ is now confirmed, and it is relatively easy to measure, its impact on performance is difficult to ascertain since in normal, healthy workers the performance variation over a 9 to 5 working day is only several percent [9], which is easily masked.

For the purpose of this work the model proposed by Spencer [19] has been identified as providing a suitable basis for a micro-model. The work was selected as it is laboratory based, it is a progression of previous work of various researchers and it has a relatively large sample size for this kind of study. In addition the model proposed is a mathematical function with easily obtained inputs. Spencer's work studied 30 students over a 9-day period of irregular spaced work and rest episodes. Based on these results and previous studies a model was developed incorporating "time of day" and "time since sleep" effects to predict performance for work episodes starting at different times of the day. The assumptions made in the construction of Spencer's model are described in detail in Section 4 of his work [19]. The performance on the task used in the study, the digital symbol substitution task (DSST) is predicted for time of day, T, and time since sleep, t, by Eq. (4.3). A typical result for a worker who wakes $3 \mathrm{~h}$ before the start of each shift is shown in Fig. 2.

$\operatorname{DSST}(T, t)=233.3+1.54 t-0.304 t^{2}+0.0108 t^{3}+4.97 \cos (2 \pi(T-17.05) / 24)$

This equation will be assumed to directly represent the variation in activity time for a worker on any assembly task. It should be noted that this equation is based on the original experimental population, and in applying it to the population in the current study we are assuming a constancy of the coefficients, with individual differences represented only in the time since sleep parameter. In order to improve the robustness of the model it would be 
necessary to conduct the experimental and analysis procedures of the original study with a sample of workers, and this is not feasible at the present time.

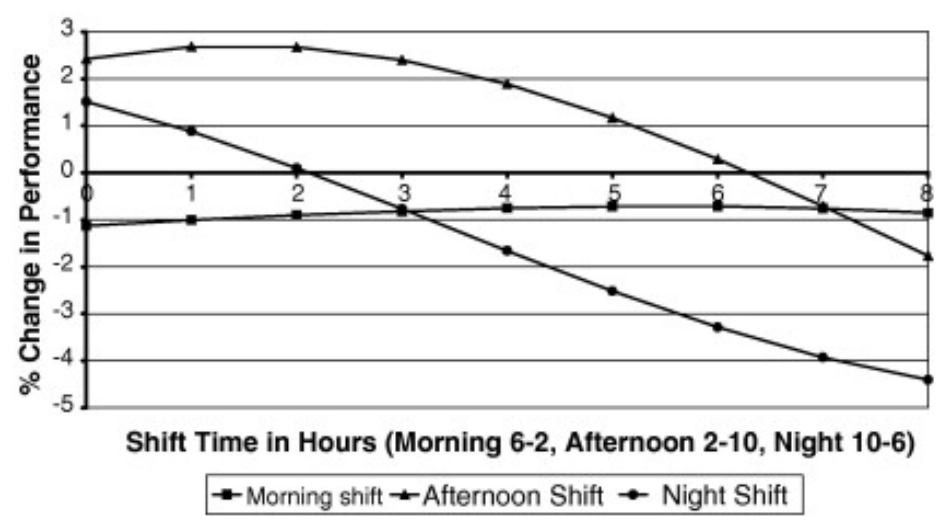

Fig. 2. - Spencer's circadian rhythm model for digital symbol substitution task (DSST): work start time $3 \mathrm{~h}$ after waking.

\section{Incorporation of human performance models into manufacturing simulation}

This section examines the feasibility of applying micro-models of human performance factors to system design.

It is a common observation that the predicted production rate of a simulation is higher than the actual output of the real system. This gap increases when looking at a simulation model of labour intensive systems like assembly lines. As it is believed that a significant proportion of the gap is due to human variation, the micro-models of human performance factors described in Section 4 will be introduced into the test simulation to examine if they have any effect on the overall system performance. At this stage of the research the absolute magnitude and accuracy of the micro-model output is not significant; rather, the intention is to find out if levels of perturbations of the form typically created by models of human performance produce significant changes in system performance. In addition, the experiments will verify that the method used to integrate human factor micro-models into DES is appropriate.

The assembly line model was constructed in Witness, a DES package widely used within the automotive industry for manufacturing system design. It has the capability to access data from Excel spreadsheets, which supports the integration of the micro-models in their current form, as initially the micro-models have been implemented in Microsoft Excel spreadsheets.

The age models require nothing more than the ages of the workers under study, while the CR model requires the normal wake time for each worker, corresponding to each shift, as well as the time of day. The output of each model is a cycle time adjustment value, as a percentage of the planned cycle time, for the workers activity time at any given time of day for any given shift pattern.

The cycle times predicted by each model were pre-calculated, and the results formatted as a lookup table. The table was then read into the Witness model as part of the simulation initialisation process. Each worker entity within the simulation model contains a short section of Witness code that selects the appropriate cycle time from the lookup table based on the hour of day and the age of the operator.

The impact of each of the models was assessed independently in order to make interpretation of the results easier. For each HPM, a number of simulation runs was performed, each for a simulated run of one year. None of the elements in the simulation were modelled stochastically. A warm-up period of one day was used to avoid recording transient system behaviour.

Individually human performance models may only improve simulation accuracy by very small increments. The accuracy of models applied in isolation is therefore limited. Combining a number of micro-models of the most significant factors affecting worker performance should provide greater accuracy. Hence, a HPM tool may consist of a number of human performance models that define how worker performance changes under different operating 
conditions, e.g. temperature, lighting, culture, motivation, personality. This modelling approach is known as "micromodelling", and the individual models are known as "micro-models" (see Fig. 3).

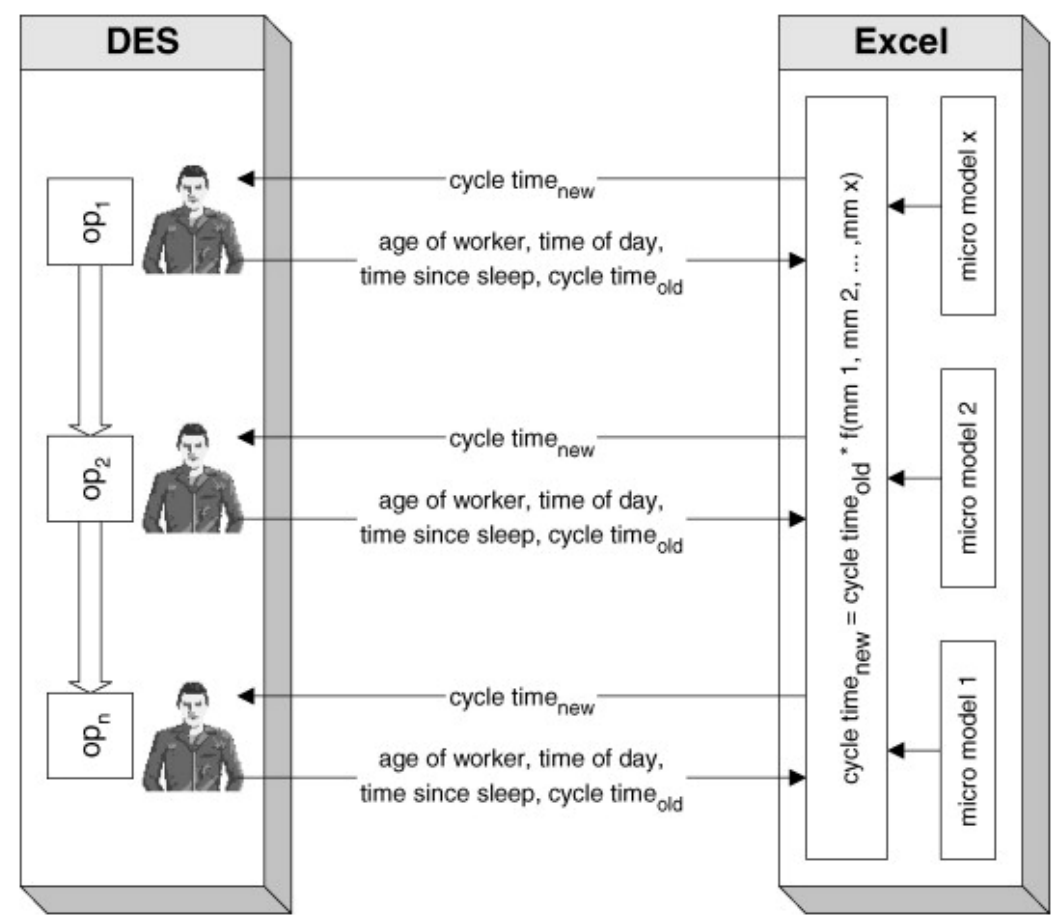

Fig. 3. Interaction of micro-models in Excel and DES labour entities.

\section{Experimental results}

The age model was run with all members of the workforce having the same age, to avoid complexity issues such as moving bottlenecks. The output of the age model for each individual worker is a single value that does not change over the period of the simulation, as shown in Table 1. The output of each run using the age model is also shown in Table 1, with the maximum output, at age 30 , coded as $100 \%$, and all other outputs expressed as percentages of the maximum.

\begin{tabular}{|l|l|l|l|}
\hline Run no. & Age (years) & Age model predicted cycle time increase (\%) & Simulation throughput (\%) \\
1 & 30 & 0 & 100 \\
2 & 45 & 6 & 97 \\
3 & 50 & 11 & 94 \\
4 & 55 & 18 & 89 \\
5 & 60 & 26 & 84 \\
6 & 65 & 35 & 78 \\
\hline
\end{tabular}

Table 1. - Results from age model tests

The CR model was driven with the wake times shown in Table 2. All members of the workforce were represented using the same wake times, once again to avoid complexity issues. The simulation output with the CR model is also shown in Table 2, coded as a percentage of the age model maximum.

\begin{tabular}{|l|l|l|l|l|}
\hline Run no. & \multicolumn{2}{|l|}{ Wake time } & \multicolumn{2}{l|}{ Simulation throughput (\%) } \\
\hline Shift 1 & Shift 2 & \multicolumn{2}{l|}{ Shift 3 } & \\
\hline 1 & $05: 30$ & $11: 00$ & $14: 00$ & 99.4 \\
2 & $05: 15$ & $10: 30$ & $13: 30$ & 99.3 \\
3 & $05: 00$ & $10: 00$ & $13: 00$ & 99.1 \\
4 & $04: 45$ & $09: 30$ & $12: 30$ & 99.0 \\
5 & $04: 30$ & $09: 00$ & $12: 00$ & 99.0 \\
6 & $04: 15$ & $08: 30$ & $11: 30$ & 98.8 \\
\hline
\end{tabular}

Table 2. - Wake times and simulation outputs for CR model 


\section{Discussion and conclusions}

The inclusion of the HPM factors clearly has an impact on the performance of the simulated production system. However, there are many issues that need further investigation. In particular, validation of the models, collection of real worker data to provide model inputs, comparison of the simulation results with the manufacturing system, and further sensitivity analysis.

The age model increases cycle times in the simulation by up to $35 \%$, and hence produces a large decrease in throughput. Since the age model output results in an equal step change increase in all the cycle times in the simulation model, it is reasonable to assume that the simulation output should be reduced by an equal amount. Fig. 4 shows the age model output and simulation outputs, and clearly demonstrates that this is not the case. The system is thus less sensitive to the age model perturbation than was expected and further experimentation is required to investigate the cause of this behaviour.

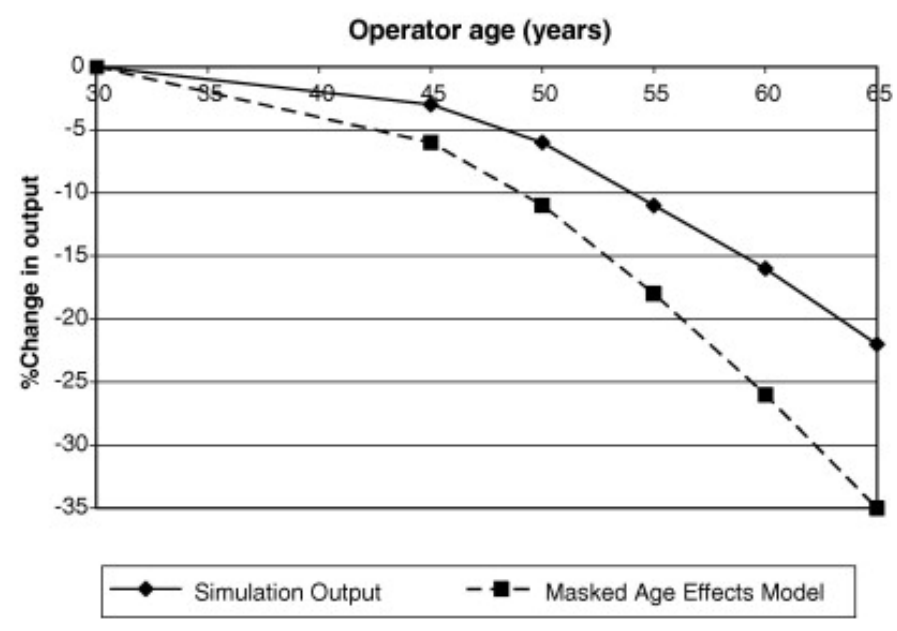

Fig. 4. Graph of changes in simulation throughput and expected throughput decrease due to age model vs. operator age.

As the CR model output is cyclic over a 24-h period, resulting in cycle time decreases as well as increases, a comparison between the CR model output and the simulation output is not feasible. The small changes in simulation output obtained with the CR model suggest that simulations are less sensitive to perturbations that are cyclic with a mean value of approximately zero. Further experimentation is required to investigate this.

Validation poses several problems when the research incorporates multiple micro-models, as it is almost impossible, in a quasi-experimental environment, to maintain stable control variables to assess the accuracy of individual micromodels. This problem is multiplied in the ethically sensitive environment of manufacturing, as much of the data required for validation would be of a personal nature, and would require the close monitoring of the performance of individual employees. One possible solution to this is to "mass" validate the micro-models, by comparing the performance of a real world system with a Witness model incorporating all appropriate micro-models.

To conclude, this work has shown that it is possible to connect external HPM tools to DES tools such as Witness, in order to introduce models of human variability. The accuracy of the resulting simulation is dependent on the validity and accuracy of the micro-models used, and hence the next stages of the research will focus on developing accurate models of appropriate human factors.

\section{References}

[1] W. Bernhardt, A. Schilling, Simulation of group work processes in manufacturing, in: Proceedings of the 1997 Winter Simulation Conference, Atlanta, Georgia, 1997, pp. 888-891

[2] L. Ehrhardt, H. Herper, H. Gebhardt, Modelling strain of manual work in manufacturing systems, in: Proceedings of the 1994 Winter Simulation Conference, Orlando, Florida, 1994, pp. 1044-1049 
[3] A. Furnham, L. Forde, K. Ferrari, Personality and work motivation, Personality and Individual Differences, 26 (1999), pp. 1035-1043

[4] C. Gaudart, Conditions for maintaining ageing operators at work-a case study conducted at an automobile manufacturing plant, Applied Ergonomics, 31 (2000), pp. 453-462.

[5] J.R. Hackman, G.R. Oldham, Motivation through the design of work: test of a theory, Organizational Behaviour and Human Performance, 16 (1976), pp. 250-279

[6] A. Jayaraman, A.K. Gunal, Application of discrete event simulation in the design of automotive powertrain manufacturing systems, in: Proceedings of the 1997 Winter Simulation Conference, Atlanta, Georgia, 1997, pp. 758764

[7] C. Klein, B. Fischer, K. Hartnegg, W.H. Heiss, M. Roth, Optomotor and neuropsychological performance in old age, Experimental Brain Research, 135 (2000), pp. 141-154

[8] The Lanner Group, WITNESS User Manual v9.0, Redditch, UK, 1998

[9] A.M. Law, W.D. Kelton, Simulation Modeling and Analysis (second ed.)McGraw-Hill, New York (1991)

[10] J.C. Marquie, J. Foret, Sleep, age and shiftwork experience, Journal of Sleep Research, 8 (1999), pp. 297-304

[11] T.H. Monk, V.C. Leng, Time of day effects in simple repetitive tasks: some possible mechanisms, Acta Psychologica, 51 (1982), pp. 207-221.

[12] D.P. Munoz, J.R. Broughton, J.E. Goldring, I.T. Armstong, Age-related performance of human subjects on saccadic eye movement tasks, Experimental Brain Research, 121 (1998), pp. 391-400

[13] R.W. Pew, A.S. Mavor, Modeling human and organizational behavior: application to military simulations, National Academy Press (1998)

[14] J. Rutenfranz, W.P. Colquhoun, P. Knauth, J.N. Ghata, Biomedical and psychosocial aspects of shift work: a review, Scandinavian Journal of Work Environment and Health, 3 (1977), pp. 165-182

[15] T.A. Salthouse, Aging and measures of processing speed, Biological Psychology, 54 (2000), pp. 35-54

[16] B. Schibye, A.F. Hansen, K. Sogaard, H. Christensen, Aerobic power and muscle strength among young and elderly workers with and without physically demanding work tasks, Applied Ergonomics, 32 (2001), pp. 425-431

[17] R.J. Shepard, Aging and productivity: some physiological issues, International Journal of Industrial Ergonomics, 25 (2000), pp. 535-545

[18] J. Snel, R. Cremer, Work and Aging: A European Perspective, Taylor and Francis (1995)

[19] M.B. Spencer, The influence of irregularity of rest and activity on performance: a model based on time since sleep and time of day, Ergonomics, 30 (9) (1987), pp. 1275-1286

[20] M.H. Stirling, K.C. Parsons, A model of human water balance, Journal of Thermal Biology, 25 (2000), pp. 187-190

[21] P. Warr, Age and job performance, Work and Aging: A European Perspective, Taylor \& Francis (1995) 